\title{
Surgical Navigation for THR: A Report on Clinical Trial Utilizing HipNav
}

\author{
Timothy J. Levison ${ }^{1}$, James E. Moody ${ }^{1}$, Branislav Jaramaz ${ }^{1,2}$, Constantinos Nikou ${ }^{1}$, \\ Anthony M. DiGioia ${ }^{1,2}$ \\ Centers for Medical Robotics and Computer Assisted Surgery, \\ ${ }^{1}$ UPMC Shadyside Hospital, Pittsburgh, PA and \\ ${ }^{2}$ Carnegie Mellon University, Pittsburgh, PA \\ $\{$ [levison, moody, branko, costa, tony] @cor.ssh.edu $\}$
}

\begin{abstract}
Computer-assisted Total Hip Replacement (THR) surgery using the HipNav surgical navigational system was evaluated. This summary reports on the first 100 HipNav clinical trial patients and focuses on: 1) patient demographics, 2) post-operative clinical outcomes, 3) incision length measurements, 4) mechanical guide measures, and 5) functional pelvic tilt measurements. Results from this clinical trial have shown no system-related complications, an improvement in post-operative clinical outcomes, reductions in soft tissue dissection, an unreliability of traditional mechanical alignment guides, and a variability of pelvic orientation during functional activity.
\end{abstract}

Keywords: orthopaedics, surgical navigation systems, clinical results

\section{INTRODUCTION}

The use of HipNav [1] computer-assisted system in total hip replacement surgery provides the ability to plan the alignment of the acetabular cup before surgery, and accurately perform the surgery according to this preoperative plan using image guided technology. We have established a methodology to evaluate the outcomes based on preoperative, intraoperative and postoperative measurements.

\section{METHODS}

The HipNav system is an image guided hip navigation system used to assist surgeons in planning of the acetabular implant placement and precise alignment according to the preoperative plan. HipNav is a collaborative research effort involving investigators from Carnegie Mellon University and UPMC Shadyside Hospital in Pittsburgh, PA. The system is composed of three components: 1) a preoperative planner; 2) a kinematic simulator; and 3) an intraoperative navigation guide. The specific technical aspects of the HipNav system have been described in other published literature $[1,2]$.

The reported results of the clinical trial involved THR patients operated with the help of the HipNav system between April 1997 and December 1999. All patients were informed of the experimental status of the system and each signed an informed consent approved by the Institutional Review Board. All patients in which the HipNav system was used were enrolled in the Total Joint Registry at UPMC Shadyside Hospital. The Total Joint Registry collects all relevant data preoperatively, 
intraoperatively and at 1, 3, 6, and 12 months post-operatively, and then annually for the rest of their lives.

\section{RESULTS}

One hundred patients have been enrolled in the study, with eight patients receiving bilateral THR procedures, for a total of 108 total hip replacement surgeries. Patient demographics are presented in Table 1.

\section{Table 1. HipNav Patient Demographics}

\begin{tabular}{|l|l|}
\hline Number of Patients & 100 patients (8 bilateral) \\
\hline Number of Procedures & 108 Hips \\
\hline Gender & 51 Female, 49 Male \\
\hline Average Age & 63 years old $(62.6)$ \\
\hline Age Range & 37 to 81 years \\
\hline Affected Hip & 48 Left, 60 Right \\
\hline
\end{tabular}

Outcome measurements were recorded using Harris Hip Score (HHS) measures[3]. The HHS measurement tool incorporates functional activity levels, perceived pain and range of motion measurements to compute a clinical outcome score. The preoperative HHS average for 100 patients was $51.6(\sigma=12.2)$. The three month HHS average was $82.1(\sigma=10.6)$. The six month HHS average was $88.5(\sigma=$ 9.8). One year postoperative HHS showed an average of $93.5(\sigma=7.9)$.

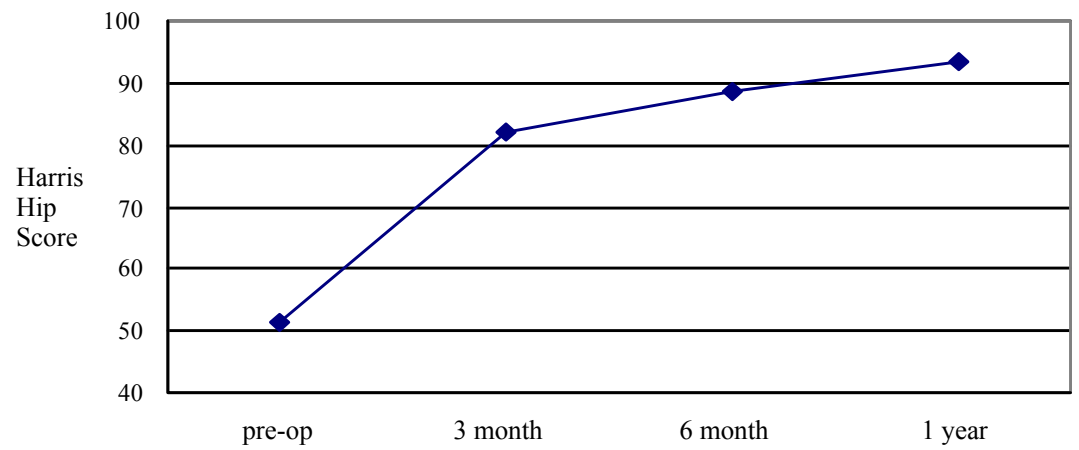

Fig 1. Change in Harris Hip Score Averages from Pre-op to 1 year Post-op following Total Hip Arthroplasty using HipNav

Results from the HipNav clinical study have also shown a reduction in the extent of soft tissue dissection on the order of 50\% when compared to traditional THR techniques. Skin incisions for all 100 patients ranged between $7.3 \mathrm{~cm}$ to $23.8 \mathrm{~cm}$, with an average length of $13.6 \mathrm{~cm}(\sigma=4.2)$. However, beginning with the 36 th patient, the surgical navigation capabilities of the HipNav system were utilized to enable the 
surgeon to use a less invasive surgical approach. As a consequence, the mean incision length changed from $19.6 \mathrm{~cm}(\sigma=2.2)$ with a range of $14.8 \mathrm{~cm}$ to $23.8 \mathrm{~cm}$ for the first 35 HipNav cases, to the average incision length of $12.1 \mathrm{~cm}(\sigma=3.1)$ with a range of $7.3 \mathrm{~cm}$ to $20.6 \mathrm{~cm}$ in patients 36 to 100 .

Before the cup was placed in the preoperatively planned orientation, acetabular component orientation using traditional mechanical guides was measured by the HipNav system. The goal of the mechanical guides is to align the acetabular cup in $45^{\circ}$ of abduction and $20^{\circ}$ of flexion. However, results of the measured cup alignment using the mechanical guide ranged from $35^{\circ}$ to $59^{\circ}$ in abduction and from $26^{\circ}$ to $33^{\circ}$ of flexion.

Functional pelvic orientation, expressed as a change in pelvic flexion and extension, was radiographically measured in all patients. These measurements of pelvic tilt for each patient represent the differences seen as they moved from a standing to a sitting position. Results of pelvic tilt measurements showed a trend towards a neutral pelvic orientation during standing, with a range of $-16^{\circ}$ to $27^{\circ}$ ( $\mu=$ $2^{\circ}, \sigma=8^{\circ}$ ). However, a significant posterior tilting was seen among patients in a sitting position, with a range of pelvic tilting from $5^{\circ}$ to $-58^{\circ}\left(\mu=-35^{\circ}, \sigma=14^{\circ}\right)$.

\section{DISCUSSION}

The HipNav system has been successfully utilized during the ongoing clinical trial and has been shown to provide a measurement tool that is useful in examining conventional methods of THR. HipNav also provides the surgeon with the ability to accurately align implants and to implement less invasive surgical procedures while not significantly increasing surgical time. The reduction in incision length, and thus soft tissue dissection, is expected to decrease complication rates and to increase the rate of recovery following surgery. HipNav patients have also shown an improvement in post-operative clinical outcomes measured by Harris Hip Scores. The current version of the system can now assist on the femoral side of the joint, permitting on-line leg length determination and patient specific maps of safe, stable range of motion.

\section{REFERENCES}

1. DiGioia AM, Jaramaz B, Blackwell M, et al: Image guided navigation system to measure intraoperatively acetabular implant alignment. Clinical Orthopaedics and Related Research, Vol. 355, pp 8-22, 1998.

2. DiGioia AM, Jaramaz B, Nikou C, et al: Surgical navigation for total hip replacement with the use of HipNav. Operative Techniques in Orthopaedics, Vol. 10, No. 1, pp 3-8, 2000.

3. Harris WH: Traumatic arthritis of the hip after dislocation and acetabular fractures: treatment by mold arthroplasty. An end-result study using a new method of result evaluation. J Bone Joint Surg 51A:737, 1969. 